\title{
Methoden der optischen Fernerkundung zur Erfassung von Bedeckungsmustern der Schneedecke als Eingangsdaten für hydrologische Eis- und Schneeschmelzmodelle
}

\author{
Optical remote-sensing methods for studying snow-cover patterns as input data
} for hydrological ice and snow-melt models

von G. Kaiser ${ }^{1}$, W. Schneider ${ }^{1}$, F. Suppan ${ }^{1}$ und M. Vollmann²

\section{Kurzfassung/Summary}

In diesem Beitrag wird nach einer Übersicht über die grundsätzlichen Möglichkeiten der optischen Fernerkundung zur Informationsgewinnung über Schnee und Eis die Anwendung eines speziell entwickelten Verfahrens für das Monitoring der Schneebedeckung mit gleichzeitig hoher räumlicher und hoher zeitlicher Auflösung beschrieben. Das Verfahren setzt voraus, dass die Ausaperung für jedes Gebiet nach einem speziellen räumlichen Muster erfolgt, das über die Jahre konstant bleibt. Dieses räumliche Muster wird aus einer Reihe hochauflösender Bilddaten vom Typ Landsat TM gewonnen, die in unterschiedlichen Jahren aufgenommen worden sein können. Der zeitliche Verlauf der Ausaperung wird aus MODIS-Bilddaten ermittelt. Damit ist es möglich, die zeitliche Entwicklung der Schneebedeckungsmuster mit einer auch für kleine Einzugsgebiete ausreichenden räumlichen Detaillierung zu erfassen.

After giving a survey of basic optical remote-sensing possibilities for obtaining information on snow and ice, this article describes a special method developed for monitoring snow cover with a high level of both spatial and temporal resolution. The method is based on the assumption that thawing proceeds to a spatial pattern specific to the particular area, and constant for major periods. This spatial pattern is studied on the basis of a series of highresolution images of type Landsat $T M$, which can date from different years. The time curve of the thawing process is determined from MODIS images. This permits recording the development of snow cover patterns with a level of resolution sufficiently high even for small catchments.

\section{Einleitung}

Die Fernerkundung erlaubt die flächendeckende Erfassung verschiedener Parameter von Schnee und Eis, die als Eingangsdaten und als Verifizierungsdaten für Schneeschmelzmodelle wichtig sind. Dieser Beitrag konzentriert sich auf die optische Fernerkundung, d.h. auf die Fernerkundung in den Spektralbereichen des sichtbaren Lichts und des Infrarots, und klammert Mikrowellenverfahren aus. Mit optischer Fernerkundung können vor allem Karten der Schneebedeckung erhalten werden. Je nach Fernerkundungssensor sind aber doch auch gewisse thematische Differenzierungen (Unterscheidung von Schnee, Firn, Eis; Gewinnung von Information über Korngrößen; Gewinnung von Information über die Verschmutzung und die damit verbundene Verringerung der Albedo der Oberflächen) möglich, und in Verbindung mit Schneeschmelzmodellen können auch Schlüsse auf den Wassergehalt des Schnees gezogen werden.

Im Gegensatz zu den meisten anderen Fernerkundungsanwendungen werden bei der Kartierung der Schneedecke Aufnahmen in kurzen Aufnahmeintervallen benötigt, um die zeitliche Entwicklung der Bedeckungsmuster zu erfassen. Dafür stehen nur wenige spezielle Fernerkundungssensoren (Sensoren mit hoher zeitlicher Auflösung) zur Verfügung, die jedoch bei realistischen Kosten nicht gleichzeitig auch eine hohe räumliche Auflösung (Detailerkennbarkeit) bieten können und daher für lokale und regionale Untersuchungen nicht oder nur bedingt geeignet sind.

In diesem Beitrag wird nach einer Übersicht über die grundsätzlichen Möglichkeiten der optischen Fernerkundung zur Informationsgewinnung über Schnee und Eis die Anwendung eines speziell entwickelten Verfahrens für das Monitoring der Schneebedeckung mit gleichzeitig hoher räumlicher und hoher zeitlicher Auflösung beschrieben. Damit ist es möglich, die zeitliche Entwicklung der Schneebedeckungsmuster mit einer auch für kleine Einzugsgebiete ausreichenden räumlichen Detaillierung zu erfassen.

\section{Verwendete Satellitenbild- daten}

Die zur Verfügung stehenden Fernerkundungssensoren unterscheiden sich aus Anwendersicht durch ihre räumliche, spektrale und zeitliche Auflösung sowie durch Verfügbarkeit und Preis. Bei der spektralen Auflösung ist zu beachten, dass für Kartierungen von Schnee und Eis Spektralbänder im sichtbaren Licht, im Nahen Infrarot und im Mittleren Infrarot gegeben sein sollen.

Im vorliegenden Projekt wurden Bilddaten der Sensoren Landsat TM und ETM+, ASTER sowie MODIS verwendet. Jeder dieser Sensoren besitzt die für die Kartierung von Schnee und Eis erforderliche spektrale Auflösung. Die Sensoren Landsat und ASTER haben Pixelgrößen zwischen 15 $\mathrm{m}$ und $30 \mathrm{~m}$. Ihre spektrale Auf- 


\begin{tabular}{|c|c|c|c|c|c|c|c|c|}
\hline \multicolumn{3}{|c|}{ Landsat TM und ETM+ } & \multicolumn{3}{|c|}{ ASTER } & \multicolumn{3}{|c|}{ MODIS } \\
\hline Band & Bereich & Pixelgr. & Band & Bereich & Pixelgr. & Band & Bereich & Pixelgr. \\
\hline 1 & $450-520 \mathrm{~nm}$ & $30 \mathrm{~m}$ & 1 & $520-600 \mathrm{~nm}$ & $15 \mathrm{~m}$ & 1 & $620-670 \mathrm{~nm}$ & $250 \mathrm{~m}$ \\
\hline 2 & $520-600 \mathrm{~nm}$ & $30 \mathrm{~m}$ & 2 & $630-690 \mathrm{~nm}$ & $15 \mathrm{~m}$ & 2 & $841-876 \mathrm{~nm}$ & $250 \mathrm{~m}$ \\
\hline 3 & $630-690 \mathrm{~nm}$ & $30 \mathrm{~m}$ & 3 & $760-860 \mathrm{~nm}$ & $15 \mathrm{~m}$ & 3 & $459-479 \mathrm{~nm}$ & $500 \mathrm{~m}$ \\
\hline 4 & $750-900 \mathrm{~nm}$ & $30 \mathrm{~m}$ & 4 & $1,60-1,70 \mu \mathrm{m}$ & $30 \mathrm{~m}$ & 4 & $545-565 \mathrm{~nm}$ & $500 \mathrm{~m}$ \\
\hline 5 & $1,55-1,75 \mu \mathrm{m}$ & $30 \mathrm{~m}$ & 5 & $2,15-2,19 \mu \mathrm{m}$ & $30 \mathrm{~m}$ & 5 & $1,23-1,25 \mu \mathrm{m}$ & $500 \mathrm{~m}$ \\
\hline 6 & $10,4-12,5 \mu \mathrm{m}$ & $60 \mathrm{~m}$ & $\ldots$ & $\ldots$ & $30 \mathrm{~m}$ & 6 & $1,63-1,65 \mu \mathrm{m}$ & $500 \mathrm{~m}$ \\
\hline \multirow[t]{2}{*}{7} & $2,09-2,35 \mu \mathrm{m}$ & $30 \mathrm{~m}$ & 10 & $8,13-8,48 \mu \mathrm{m}$ & $90 \mathrm{~m}$ & 7 & $2,11-2,16 \mu \mathrm{m}$ & $500 \mathrm{~m}$ \\
\hline & & & $\ldots$ & $\ldots$ & $90 \mathrm{~m}$ & $\ldots$ & $\ldots$ & \\
\hline
\end{tabular}

lösung ist durch 7 bis 14 Spektralbänder im sichtbaren Licht und im Nahen, im Mittleren und im Thermischen Infrarot gekennzeichnet. Die zeitliche Auflösung ist jedoch sehr bescheiden: In Österreich kann etwa bei Landsat mit nur wenigen hinreichend wolkenfreien Bildern pro Jahr gerechnet werden. Die zeitliche Verfolgung von Ausaperungsmustern ist damit nicht möglich.

Der Sensor MODIS, der in zwei Exemplaren auf den Satelliten Terra und Aqua im Erdumlauf ist, kann demgegenüber fast täglich jeden beliebigen Punkt der Erdoberfläche aufnehmen, sodass sich auch eine große Zahl an wolkenfreien Bildern ergibt. Bei einer Flughöhe von $705 \mathrm{~km}$ und einer Streifenbreite von $2330 \mathrm{~km}$ werden die randnahen Bereiche allerdings mit sehr schräger Blickrichtung aufgenommen. Es werden 36 Spektralbänder in allen Bereichen des optischen Spektrums erfasst, allerdings mit Pixelgrößen von bestenfalls (bei lotrechter Blickrichtung) $250 \mathrm{~m}$ bis $1 \mathrm{~km}$. Diese Bilddaten sind gratis zu beziehen.

Die Spektralbänder von Landsat, ASTER und MODIS sind in Tabelle 1 aufgelistet.

\section{Schnee, Firn und Eis in multispektralen Fernerkun- dungsbildern}

Die spektrale Reflexion von Schnee ist gekennzeichnet durch
- eine sehr hohe Reflexion (nahe $100 \%$ ) im Bereich des sichtbaren Lichts, wobei verschmutzte Oberflächen auch kleinere Reflexionswerte zeigen können,

- eine geringere Reflexion im Bereich des Nahen Infrarot (Wellenlänge 0,7 bis $1,4 \mu \mathrm{m}$ ), wobei die Streubreite groß ist (höherer Reflexionsgrad für kleine Korngrößen $(0,05 \mathrm{~mm})$ und niedrigerer Reflexionsgrad für große Korngrößen (1,00 mm)),

- verschwindend kleine Reflexion in den Wasserabsorptionsbanden (um 1,5 $\mu \mathrm{m}, 2,0 \mu \mathrm{m}$ and 2,5 $\mu \mathrm{m})$, und

- niedrige Reflexion im Mittleren Infrarot außerhalb dieser Wasserabsorptionsbanden, wobei der Reflexionsgrad so wie im $\mathrm{Na}$ hen Infrarot von der Korngröße abhängt.

Die spektrale Reflexion von Eis zeigt ähnliche Trends, mit

- geringerer Reflexion (etwa 50\%) für sichtbares Licht,

- bedeutend geringerer Reflexion (etwa 10\%) im Nahen Infrarot, und

- Reflexionsgrad Null im Mittleren Infrarot.

Verunreinigungen können den Reflexionsgrad von Gletschereis im sichtbaren Licht stark verringern (bis unter $20 \%$ ) und im Mittleren Infrarot außerhalb der Wasserabsorptionsbanden erhöhen.
Diagramme, die diese Reflexionscharakteristiken illustrieren, finden sich z. B. bei [Hall et al., 1985].

Bei der Kartierung von Schnee und Eis aus optischen Fernerkundungsbilddaten wird meist der NDSI (Normalised Difference Snow Index) verwendet, der als normalisierte Differenz der Reflexionsgradwerte in jenen Wellenlängenbereichen definiert ist, in denen die Reflexion von Schnee ein Maximum bzw. ein Minimum hat:

$$
N D S I=\frac{\rho_{G}-\rho_{M I R}}{\rho_{G}+\rho_{M I R}} .
$$

Hier kennzeichnet G den Spektralbereich Grün und MIR den Spektralbereich Mittleres Infrarot. Oft werden die Reflexionsgradwerte $\rho$ einfach durch die Pixelwerte eines radiometrisch nicht kalibrierten Bildes in den entsprechenden Spektralbereichen ersetzt, obwohl man dann etwas andere Werte als nach der exakten Definition erhält. Für Landsat-Bilddaten und für ASTER-Bilddaten gilt dann z. B.

$$
\begin{aligned}
& N D S I=\frac{T M 2-T M 5}{T M 2+T M 5} \quad \text { bzw. } \\
& N D S I=\frac{A S T 1-A S T 4}{A S T 1+A S T 4}
\end{aligned}
$$

wobei TMi der Pixelwert im Landsat-TM-Band i und ASTi der Pixelwert im ASTER- Band i ist. 
Der Grenzwert für die Erkennung von Schnee und Eis liegt bei etwa 0,4 : NDSI $>0,4$ ist Schnee oder Eis, NDSI $<0,4$ eine andere Oberfläche. Probleme ergeben sich jedoch oft bei Gewässeroberflächen, die ebenfalls einen hohen NDSI zeigen können. Für diesen Fall wird daher eine zweite Bedingung verwendet: Die Reflexion im Nahen Infrarot ist für Gewässer niedrig, für Schnee und Eis höher. Der Schwellenwert dafür liegt bei Landsat (TM4) bei einem Pixelwert, der einem Reflexionsgrad von etwa $11 \%$ entspricht.

Sättigungseffekte von Schneeflächen in Satellitenbilddaten in den Spektralbändern des sichtbaren Bereichs können spezielle Probleme verursachen und müssen besonders berücksichtigt werden.

\section{Kartierung von Schnee, Firn und Eis aus Landsat- und ASTER-Bildern}

Im vorliegenden Projekt wurden als Grundlage Kartierungen mit Landsat- und ASTER-Bilddaten durchgeführt, die dann in einem weiteren Schritt mit MODIS-Auswertungen kombiniert wurden (siehe Kapitel 6).

Der Arbeitsablauf für die Kartierung von Schnee und Eis aus Landsat- und ASTER-Bildern umfasst die folgenden Schritte:

- Georeferenzierung

- Luftlichtkorrektur

- Herstellung einer Maske für Schnee und Eis

- radiometrisch-topografische Normalisierung der Bilddaten, optimiert auf Schnee und Eis

- Klassifizierung von Schnee und Eis in die Kategorien Neuschnee, Firn und Nassschnee, Gletschereis.

Soweit die Bilddaten nicht schon mit ausreichender Genauigkeit georeferenziert vorliegen, ist eine Entzerrung unter Verwendung eines digitalen Geländehöhenmodells durchzuführen. Dabei wird eine Genauigkeit erreicht, die durch einen mittleren Fehler von etwa 0,5 bis 1 Pixel gekennzeichnet ist, also bei Landsat durch einen Fehler von $15 \mathrm{~m}$ bis $30 \mathrm{~m}$.

Der Einfluss des Luftlichts (einfallende Sonnenstrahlung, die in der Atmosphäre in Richtung zum Sensor gestreut wird und als additiver Term die Pixelwerte verfälscht) ist im Hochgebirge meist klein. Das Luftlicht wurde im vorliegenden Projekt mit der einfachen „darkobject-subtraction“-Methode korrigiert: Für jedes Spektralband wird dabei angenommen, dass der niedrigste Pixelwert, der im ganzen Bild beobachtet wird, eine Oberfläche mit verschwindend kleinem Reflexionsgrad darstellt und daher die Größe des Luftlichts angibt (die bei dieser Methode als über das gesamte Bild konstant angenommen wird). Dieser Wert wird von allen Pixeln des Bildes subtrahiert.

Die Maske für Schnee und Eis wurde unter Verwendung des NDSI und des zusätzlichen Schwellenwertes für den Pixelwert im Nahen Infrarot zur Berücksichtigung von Gewässeroberflächen (siehe Kapitel 3) hergestellt.

Die radiometrische-topografische Normalisierung soll die Pixelwerte gleichartiger Oberflächen unabhängig von der Geländeneigung und damit unabhängig von der Intensität der Sonneneinstrahlung auf ein einheitliches Niveau bringen, also z. B. die Helligkeit von Nordhängen und von Südhängen aneinander angleichen. Für diesen Zweck wurde die sogenannte CKorrektur verwendet. Diese Methode setzt Lambert'sche Oberflächen voraus (Oberflächen mit einer von der Beobachtungsrichtung unabhängigen Strahldichte, das heißt diffus reflektierende Oberflächen - eine Bedingung, die für Schnee und Eis in hinreichender Näherung erfüllt ist). Die Einstrahlung wird aus zwei Komponenten bestehend angenommen: aus gerichteter Sonnenstrahlung, und aus diffuser, aus allen Richtungen des Halbraums kommender Himmelsstrahlung. Das Verhältnis dieser beiden Komponenten wird aus dem Bild selbst mit einer Regressionsmethode abgeschätzt. Dazu werden aus dem Bild abgelesene Pixelwerte in einem Diagramm gegen den Kosinus des Einstrahlwinkels der Sonne auf das (geneigte) Gelände an der betreffenden Stelle aufgetragen. Dieser Einstrahlwinkel kann bei bekanntem Sonnenstand mit Hilfe eines digitalen Geländehöhenmodells für jedes Pixel ermittelt werden. Aus den Regressionsparametern kann dann das Verhältnis von Sonnenstrahlung und Himmelsstrahlung für jedes Pixel und damit der Einfluss der Geländeneigung berechnet und korrigiert werden (Umrechnung auf fiktives ebenes Gelände). Details zu dieser Korrekturmethode sind z. B. aus [Teillet et al., 1982] zu ersehen.

Bei der C-Korrektur-Methode muss vorausgesetzt werden, dass für die Regression Pixelwerte von Oberflächen mit identischen Reflexionseigenschaften aus dem Bild abgelesen werden. Es darf insbesondere keine Abhängigkeit der Reflexionseigenschaften von Neigung und Exposition geben. Eine Vorbedingung dafür ist auf jeden Fall, dass diese Pixelwerte ausschließlich von Schneeflächen genommen werden. Diese werden mit Hilfe der Schneemaske identifiziert. Die Bedingung gleichförmiger Oberflächeneigenschaften für die verwendeten Pixelwerte kann trotzdem nicht garantiert werden, sodass es sich bei dieser Form der Normalisierung nur um ein Näherungsverfahren handeln kann. Ein zweiter Grund für Ungenauigkeiten liegt in der Vernachlässigung der Abschattung der Himmelsstrahlung aus Teilen des Halbraums durch höherliegende Geländeteile. Weiters würde man für die Herstellung der Schneemaske selbst schon ein radiometrisch-topografisch normalisiertes Bild benötigen. Da diese 

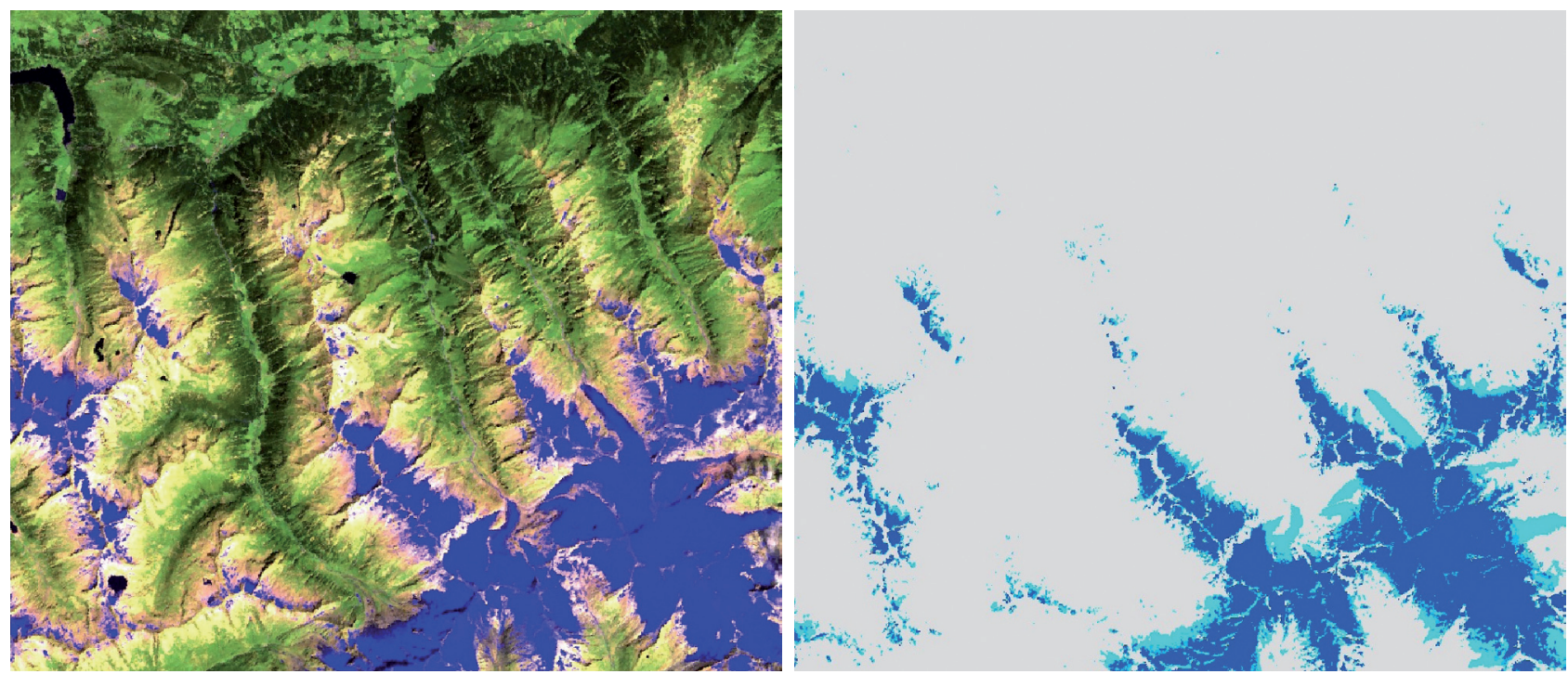

Abb. 1: Testgebiet Mittersill: Bildausschnitt von Landsat ETM+ (Spektralbänder 7-5-2 in R-G-B) vom 28.7.2002 und daraus abgeleitete Karte der Schnee- und Eisflächen (Schnee: dunkelblau, Eis: türkis).
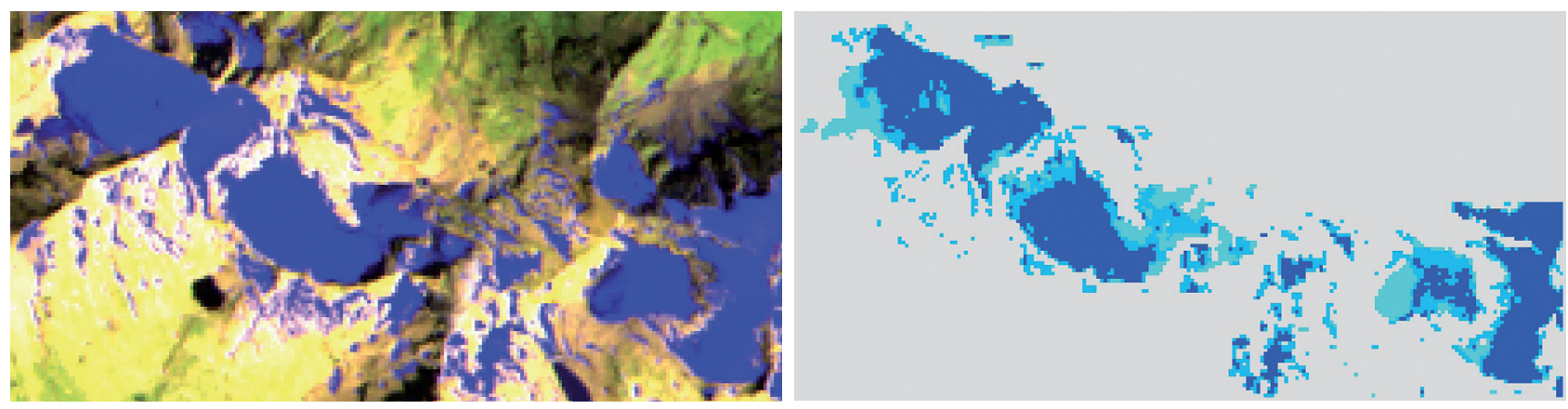

Abb. 2: Testgebiet Goldberg-Gruppe: Bildausschnitt von Landsat TM (Spektralbänder 7-5-2 in R-G-B) vom 12.8.1993 und daraus abgeleitete Karte der Schnee- und Eisflächen (Schnee: dunkelblau, Firn: hellblau, Eis: türkis).

Normalisierung erst anschließend durchgeführt wird, kann es sich auch aus diesem Grund nur um ein Näherungsverfahren handeln.

Die abschließende Klassifizierung könnte im Prinzip mit Methoden der statistischen überwachten Klassifizierung unter Verwendung von „Trainingsgebieten“ durchgeführt werden, mit denen man die zu unterscheidenden Kategorien (Neuschnee, Firn und Nassschnee, Gletschereis) definiert und charakterisiert. Für den Zeitpunkt der Aufnahme der Fernerkundungsbilder waren verlässliche Informationen über solche Trainingsgebiete jedoch nicht verfügbar. Deshalb wurde ein wissensbasierter Ansatz gewählt. Grundlage war die Tatsache, dass der Reflexions- grad von Schnee im Bereich des Nahen Infrarot mit der Korngröße des Schnees abnimmt, und dass der Reflexionsgrad von Gletschereis in diesem Spektralbereich noch niedriger ist als jener von Schnee mit maximaler Korngröße. Es wurde daher eine einfache Klassifikation allein unter Verwendung des Nahen Infrarot durchgeführt. Die Schwellenwerte zur Unterscheidung der Kategorien wurden aus den in der Literatur veröffentlichten Reflexionskurven sowie aus $\mathrm{Pi}$ xelwerten von Bildbereichen erhalten, die visuell interpretiert werden konnten. Es ergaben sich folgende Mittelwerte $\left(\rho_{\mathrm{s}}\right.$ ist der höchste an Schneeflächen beobachtete Reflexionswert, der Neuschnee zugeordnet wird):
Neuschnee:

$\rho / \rho_{s}=1,00$

(definitionsgemäß)

Firn, Nassschnee: $\quad \rho / \rho_{s}=0,67$ Gletschereis: $\quad \rho / \rho_{s}=0,40$

Aus diesen Mittelwerten wurden dann die Schwellenwerte bestimmt.

Die Abbildungen 1 und 2 zeigen Landsat-Bildausschnitte und die daraus abgeleiteten Kartierungsergebnisse für das Testgebiet Mittersill und das Testgebiet Goldberggruppe.

5. Kartierung von Schnee und Eis aus MODIS-Bildern: Standard-MODIS-Datenprodukte

Aus den MODIS-Bilddaten werden routinemäßig sogenannte MODIS- 
Datenprodukte [GSFC, 2008] erzeugt, unter denen sich auch das „MODIS Snow Cover“ - Produkt befindet. Schneebedeckung und Schnee-Albedo werden bei Pixelgrößen von $500 \mathrm{~m}$ und 0,05 Grad (entsprechend 5,5 km am Äquator) täglich zur Verfügung gestellt. Auch die partielle Schneebedeckung innerhalb eines Pixels wird dargestellt. Aus diesen Kartierungen werden auch Versionen mit 8-Tages- und Monats-Mittelwerten der Schneebedeckung hergestellt [NSIDC 2008], [Riggs et al., 2006].

Die Ausweisung eines Pixels als schneebedeckt bzw. nicht schneebedeckt basiert auf einem Algorithmus ähnlich jenem, der in Abschnitt 3 beschrieben wurde. Ein Pixel wird als schneebedeckt identifiziert, wenn der Normalised Difference Snow Index NDSI > 0.4 und wenn der Reflexionsgrad im Nahen Infrarot (Band 2, 841-876 nm) mindestens $11 \%$ ist. Zusätzlich wird mit der Forderung eines Reflexionsgrades von mindestens $10 \%$ in Band 4 (545-565 nm) verhindert, dass Pixel von sehr dunklen Geländebereichen, wie etwa dichten $\mathrm{Na}$ delwäldern, fälschlicherweise als Schnee markiert werden, weil die Berechnung des NDSI bei einem ungünstigen Signal/Rausch-Verhältnis sehr unsicher wird [Klein et al., 1998]. Zur weiteren Reduktion von Falsch-Klassifikationen werden eine Wolken-Maske, der NDVI und ein Temperaturfilter eingesetzt. Der NDVI wird verwendet, um die Schneekartierung im Wald zu verbessern [Klein et al., 1998]. Mit dem Temperaturfilter wird erreicht, dass helle warme Oberflächen (z. B. Fels) nicht als Schnee kartiert werden.

Die Berechnung der schneebedeckten Teilfläche erfolgt mit einer Regressionsgleichung, die durch Vergleich von MODIS-Pixelwerten mit Landsat-Schnee-Kartierungen in unterschiedlichen Regionen entwickelt wurde [Salomonson et al., 2004].
Aus geometrischer Sicht ist zu bemerken, dass die MODIS-Datenprodukte durch Abfüllen („Gridding“) der Original-MODIS-Pixel auf die Zellen eines vorgegebenen Rasters auf der Erdoberfläche (in Sinusoidal-Projektion) erzeugt werden. Dieses Abfüllen entspricht einer Nearest-Neighbour-Interpolation, dh. die exakte Aufnahmegeometrie bzw. Information über das exakte Sichtfeld (Ground Instantaneous Field Of View, GIFOV) der einzelnen Pixel geht dadurch verloren. Eine Ausnahme stellt das sogenannte Swath-Produkt (MOD10_ L2) dar, das die Rasterdaten in der originalen Aufnahmegeometrie ohne jede Veränderung durch Projektions- und Interpolationsverfahren zur Verfügung stellt. Geometrieinformation wird dabei explizit in Form der Koordinaten einzelner Pixel beigefügt.

Genauere Methoden der Georeferenzierung werden im nächsten Abschnitt behandelt.

\section{Kartierung von Ausape- rungsmustern aus MODIS- Bildaten mit Subpixel-Auf- lösung}

\subsection{MODIS Geolocation}

Der Einfluss von Ungenauigkeiten in der Verortung der Fernerkundungsdaten auf das Abflussmodell hängt von der Größe des Einzugsgebietes bzw. von der gewählten Zellengröße ab: Je kleinräumlicher das Einzugsgebiet und je kleiner die Zellengröße des Abflussmodells sind, desto größere Anforderungen werden an die geometrische Genauigkeit der Schneekartierung gestellt. Die durch „Gridding“ (Nearest Neighbour-Resampling) hergestellten Standard-MODIS-Schneeprodukte sind vor allem für regionale bis globale Analysen geeignet. Das bis vor kurzem nicht erhältliche MOD10_L2 Swath-Produkt (siehe Abschnitt 5) basiert zwar auf der ursprünglichen Aufnahmegeometrie, enthält aber Information zur Geokodierung in einem $5 \mathrm{~km}$-Raster, d.h. die Lageinformation ist jeweils nur für jedes zehnte (in Reihenund Spaltenrichtung) 500m Pixel gegeben.

Im vorliegenden Projekt wurde deshalb ein eigener Ansatz gewählt: Statt die Standard-MODIS-Schneeprodukte zu verwenden, wurde die Schneekartierung basierend auf dem NDSI (gemäß [Hall et al., 2001]) aus den Originaldaten (Strahldichte aus dem Produkt MOD02HKM, Level-1B Calibrated Geolocated Radiances, 500m Pixelgröße) [GSFC, 2008] erstellt. Die exakte Lage und Größe der Fläche jedes Pixels am Boden (GIFOV) wurde aus der in MOD02 enthaltenen GeolocationInformation berechnet, die aus den geografischen Koordinaten des $\mathrm{Pi}$ xelzentrums jedes 1000m-Pixels besteht. Diese Koordinaten werden aus dem Schnitt der Abbildungsstrahlen (aus Satellitenposition, BlickrichtungundSensorgeometrie) mit einem digitalen Geländemodell ermittelt. Da die Schneekartierung in einer räumlichen Auflösung von $500 \mathrm{~m}$ verwendet wird, müssen die Pixelkoordinaten der 500m-Pixel durch Inter- und (an den Scangrenzen) durch Extrapolation aus dem 1000m-Koordinatenraster berechnet werden. Dieses Inter- bzw. Extrapolationsverfahren liefert die Eckpunktkoordinaten der Fläche jedes einzelnen MODIS-Pixels am Boden. Dieser Ansatz ermöglicht es auch, die durch schiefe Blickwinkel und die Topografie hervorgerufenen Verzerrungen zu berücksichtigen: Das GIFOV eines Pixels ist im allgemeinen Fall kein Quadrat, sondern ein Viereck, und es ist über die Fläche des Bildes nicht konstant (siehe Abb. 3).

\subsection{Räumliche Subpixelanalyse}

Die räumliche Subpixelanalyse [Schneider, 1993] hat die Erkennung der räumlichen Struktur der Landbedeckung auf Subpixel- 


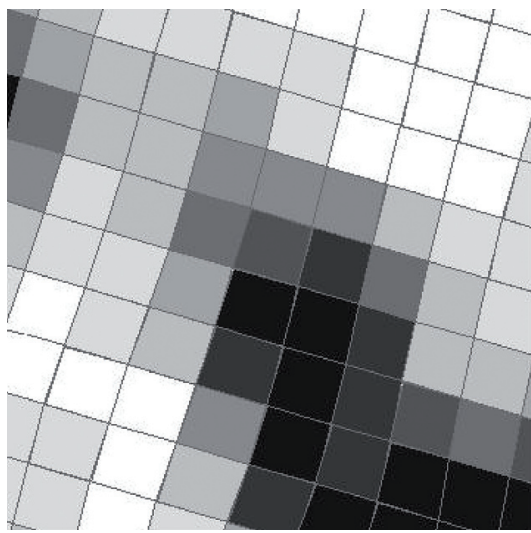

Abb. 3: Die exakten Sichtfelder (GIFOVs) der einzelnen MODIS-Pixel sind keine Quadrate, sondern allgemeine Vierecke.

\section{Niveau zum Ziel.}

Die Methode basiert auf den Pixelwerten eines Bildes und deren räumlicher Anordnung. Die vom Fernerkundungssensor abgebildeten Objekte auf der Erdoberfläche werden in diesem Verfahren lokal, d.h. für ein bestimmtes Pixel und dessen Umgebung, durch ein geometrisch-physikalisches Modell beschrieben. Dieses Modell besteht aus einem Parametersatz, der einerseits die Objektgeometrie und anderseits die "reinen“ spektralen Eigenschaften (Signaturen) des Objektes beschreibt. Die Methode wurde am Institut für Vermessung, Fernerkundung und Landinformation an der BOKU ursprünglich für landwirtschaftlich genutzte Flächen entwickelt. Dabei wird die Objektgeometrie durch Äcker beschrieben, die in geradlinigen Grenzen aneinander stoßen. Abbildung 4 zeigt beispielhaft ein geometrisches Modell zweier benachbarter Äcker mit jeweils homogenen spektralen Eigenschaften. Dieses Modell kann durch 2 geometrische Parameter (Ursprung und Orientierung der Grenzlinie) und 2 spektrale Parameter pro Bildkanal, nämlich die reinen Spektraleigenschaften der Flächen, beschrieben werden.

Im Falle der Schneekartierung muss ein anderes geometrisches Modell verwendet werden, da hier
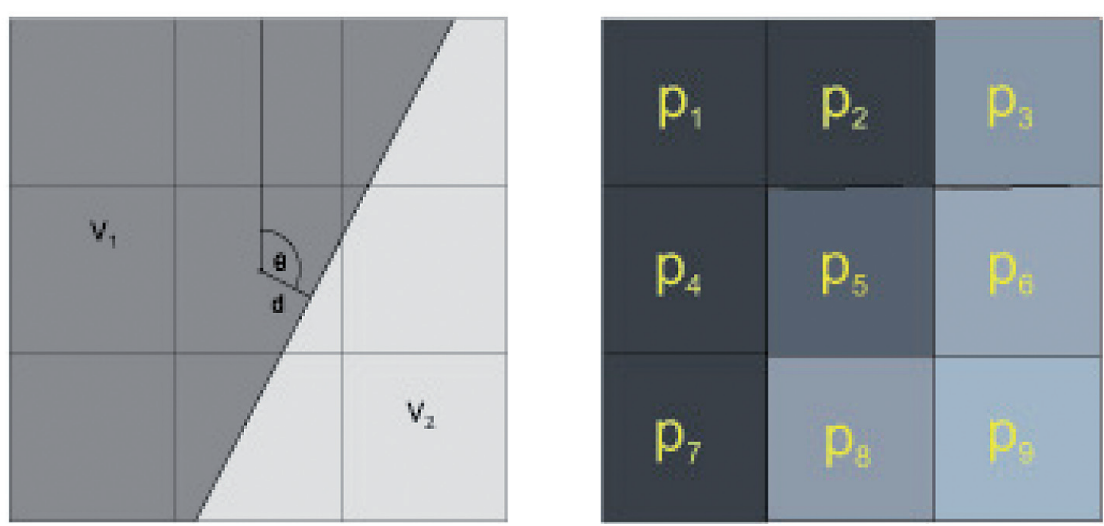

Abb. 4: links: Subpixelmodell für die Landnutzung in landwirtschaftlichen Gebieten: geometrische Parameter $\mathrm{d}, \theta$, radiometrische Parameter $\mathrm{v}_{1}, \mathrm{v}_{2}$; rechts: Pixelwerte, aus denen diese 4 Parameter geschätzt werden

keine geraden Grenzlinien erwartet werden können. Das betreffende Modell wird in Abschnitt 6.4 beschrieben.

Bei der räumlichen SubpixelAnalyse werden nun die Parameter der unterschiedlichen Modelle für jeweils ein Pixel und dessen Umgebung (bspw. ein 3x3 Fenster, siehe Abb. 4) aus den Bilddaten ermittelt. Es werden jenes Modell und jener zugehörige Parametersatz ausgewählt, welche bei Simulation des Abbildungsprozesses am ehesten die tatsächlich beobachtete Verteilung der Pixelwerte in dieser Umgebung ergeben. Ein Maximalwert der erlaubten Abweichung darf dabei nicht überschritten werden. Nach der Ermittlung des Modells und seiner Parameter kann das Bild innerhalb der betrachteten Umgebung durch Resampling auf eine feinere räumliche Auflösung umgerechnet werden.

Notwendige Voraussetzung für das Funktionieren der räumlichen Subpixel-Analyse ist die mathematische Formulierung des Abbildungsprozesses, der für ein gegebenes Objekt auf der Erdoberfläche (d.h. für die entsprechende räumliche Verteilung der spektralen Strahldichte) eine bestimmte Pixelverteilung generiert. Die einfachste Annahme ist, dass jeder Grauwert durch Integration der Objektstrahlung über ein Quadrat
- entsprechend der Oberfläche eines Detektorelementes - an der Pixelposition entsteht.

Diese Annahme ist eine Vereinfachung, die im folgenden $\mathrm{Ab}$ schnitt erweitert wird.

\subsection{Einfluss der Point Spread Function}

Auch die beste Sensoroptik bildet das Objekt nicht scharf auf die Detektorelemente ab, sondern verursacht eine mehr oder weniger starke Verwischung in der Strahlungsverteilung. Diese Unschärfe wird durch Integration der Energiedichte über die Oberfläche des Detektorelementes in das Bild übertragen. Zusätzliche Verzerrungen entstehen durch die Bewegung der Sensorplattform während der Aufnahme und durch elektronische Filter. Die genannten Effekte werden durch die sog. Point Spread Function (PSF) des Sensors [Schowengerdt, 1997] beschrieben. Weiters entspricht der quadratischen Fläche eines Detektorelementes in der Regel kein quadratischer Ausschnitt der Erdoberfläche (siehe Abschnitt 6.1). Diese Unregelmäßigkeit sollte bei der Berechnung der StrahldichteVerteilung berücksichtigt werden.

Die Point Spread Function (PSF) eines Sensors beschreibt das Bild eines infinitesimal kleinen punkt- 


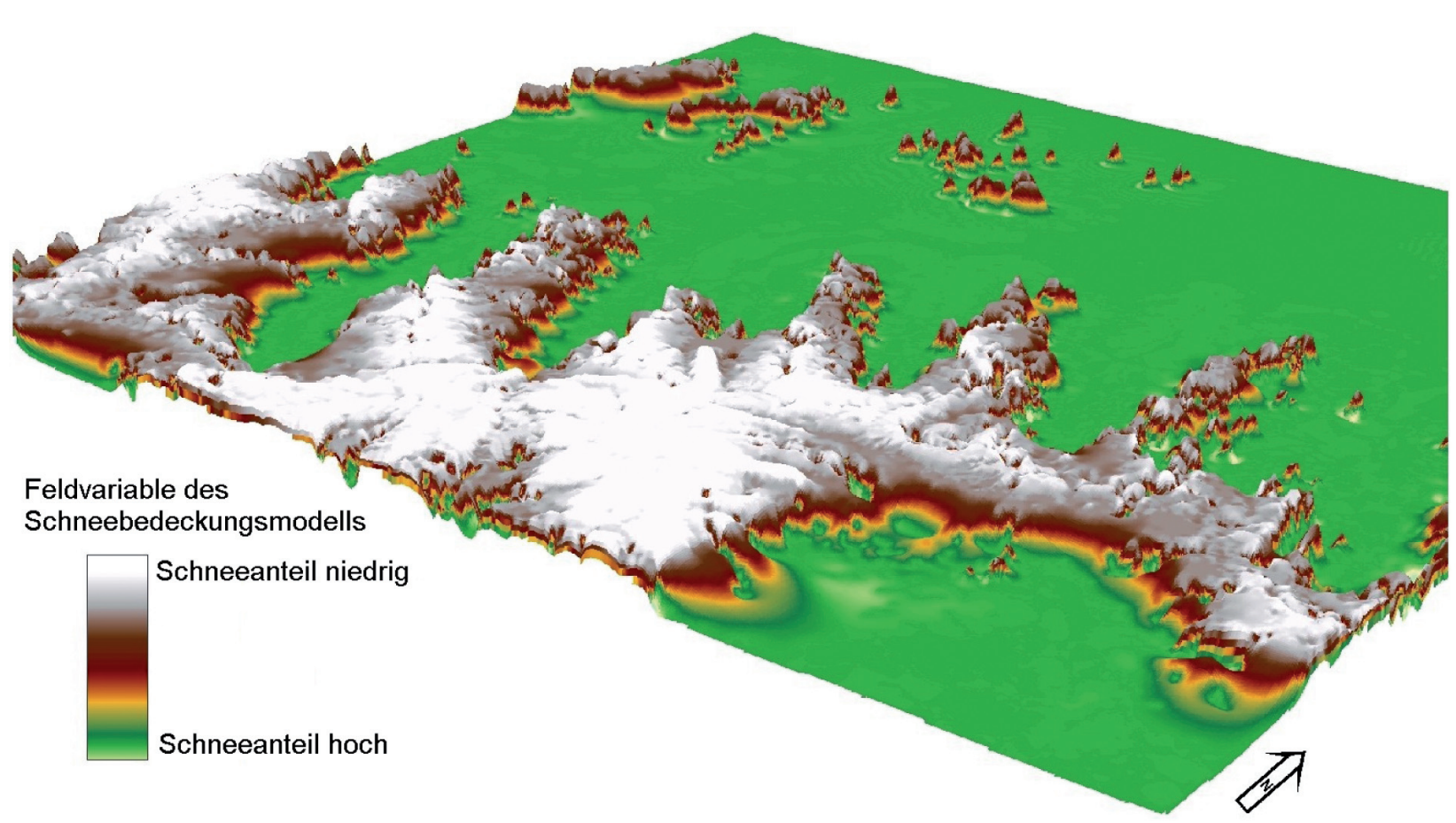

Abb. 5: Schneebedeckungsmodell in 3D-Darstellung, Testgebiet Mittersill.

förmigen Objektes, so wie es vom Abbildungssystem des Instruments erzeugt wird. Das Abbild eines realen Objektes entspricht dann der Summierung von räumlich verteilten, mit der Objektintensität gewichteten PSFs. Die Berücksichtigung der PSF bei der räumlichen Subpixelanalyse ist in [Kaiser et al., 2007] näher beschrieben.

\subsection{Kartierung der Ausape- rungsmuster}

Es wurde versucht, mit der Methode der räumlichen Subpixelanalyse unter Berücksichtigung der PSF aus MODIS-Bildern räumlich hochauflösende Schneekartierungen herzustellen. Dazu wurde zunächst der Anteil der schneebedeckten Fläche innerhalb jedes einzelnen der auf der Geländeoberfläche exakt lokalisierten PixelPolygone (siehe Abschnitt 6.1) mit der in Abschnitt 5 beschriebenen Methode ermittelt. Weiters wurde ein geometrisches Modell des Schneebedeckungs-Musters erstellt, das für eine beliebige Fläche (z. B. ein MODIS-Pixel-Polygon) die Verteilung der Schneebedeckung als Funktion eines einzigen Parameters (des SchneeflächenAnteils) ausdrückt. Dieses Modell der Schneebedeckung ist kein einfaches geometrisches Modell (wie z.B. jenes für landwirtschaftliche Flächen, in Abschnitt 6.2 dargestellt), sondern weist sehr komplexe Formen auf.

Dem Modell liegt die Annahme zugrunde, dass die räumlichen Muster der Ausaperung konstant sind: Legt man alle beobachteten Schneebedeckungsmuster eines Gebietes übereinander, so erhält man eine Schar von Umrisslinien schneebedeckter Flächen. In dieser Schar kommen keine einander schneidende Umrisslinien vor. Diese Linienschar kann in Analogie zu Höhenschichtlinien als Schar von Isolinien eines Feldes gedeutet werden, dessen Feldvariable bei geeigneter Skalierung für jeden Punkt jenen Anteil der Schneefläche an der Gesamtfläche angibt, bei dem an diesem Punkt der Schnee verschwindet.

Dieses Schneebedeckungsmodell kann daher in Analogie zu einem Geländemodell mit den entsprechenden GIS-Funktionen hergestellt werden: Aus einer Reihe hochauflösender Bilddaten (Landsat TM und Aster) werden zunächst die Schneeflächen kartiert. Die Kategorien Schnee, Firn und Eis werden zwecks Vereinfachung zusammengefasst, sodass binäre Bilder (Schnee-Nichtschnee) entstehen. Daraus werden die Umrisslinien der Schneeflächen erzeugt. Allen einem bestimmten Ausaperungsgrad entsprechenden Umrisslinien wird als Attribut der Anteil der Schneefläche an der Gesamtfläche $\mathrm{zu}$ diesem Ausaperungszeitpunkt beigefügt. Die gesamte Schar von Umrisslinien wird nun als Isolinienschar in Analogie zu Höhenschichtlinien behandelt, wobei das Attribut „Schneeflächenanteil" der Geländehöhe entspricht. Mit geeigneten GIS-Funktionen wird ein kontinuierliches Feld erzeugt. Dieses kontinuierliche Feld kann als 3D-Modell mit der Feldvariablen (Schneeflächenanteil) in der dritten Dimension dargestellt werden - siehe Abbildung 5. Man schneidet nun dieses 3D- 


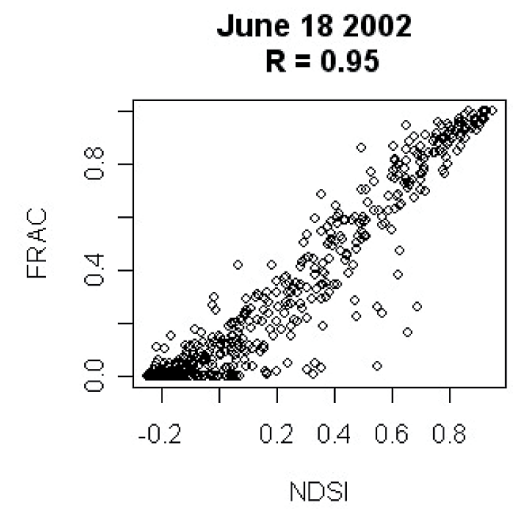

September 032004 $\mathbf{R}=\mathbf{0 . 9 5}$

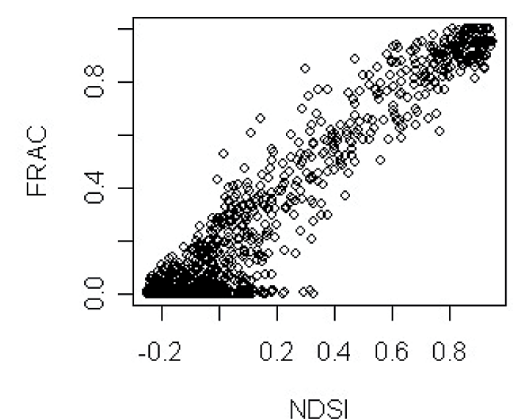

Abb. 6: Korrelation zwischen NDSI (aus MODIS mit exakter Geolocation unter Berücksichtigung der PSF) und Schneeflächenanteil (FRAC) (aus Landsat- und ASTER-Satellitenbildern) für das Testgebiet Mittersill.

September 10 2004, MOD09 $\mathbf{R}=\mathbf{0 . 8 4}$

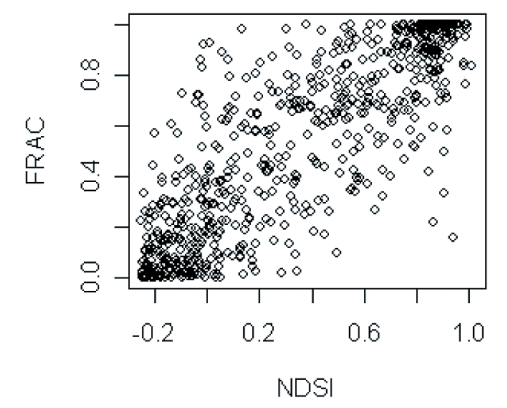

Abb. 7: Korrelation zwischen NDSI (aus MODIS Standard Product) und Schneeflächenanteil (FRAC) (aus Landsat- und ASTERSatellitenbildern) für das Testgebiet Mittersill, vergleiche mit letztem Diagramm in Abb. 6.

Modell in verschiedenen Höhen mit einer horizontalen Ebene. Jeder Schnitt stellt ein Ausaperungsmuster dar, das einem der ursprünglichen auf den hochauflösenden Satellitenbildern gegebenen Muster entspricht bzw. aus diesen durch Interpolation
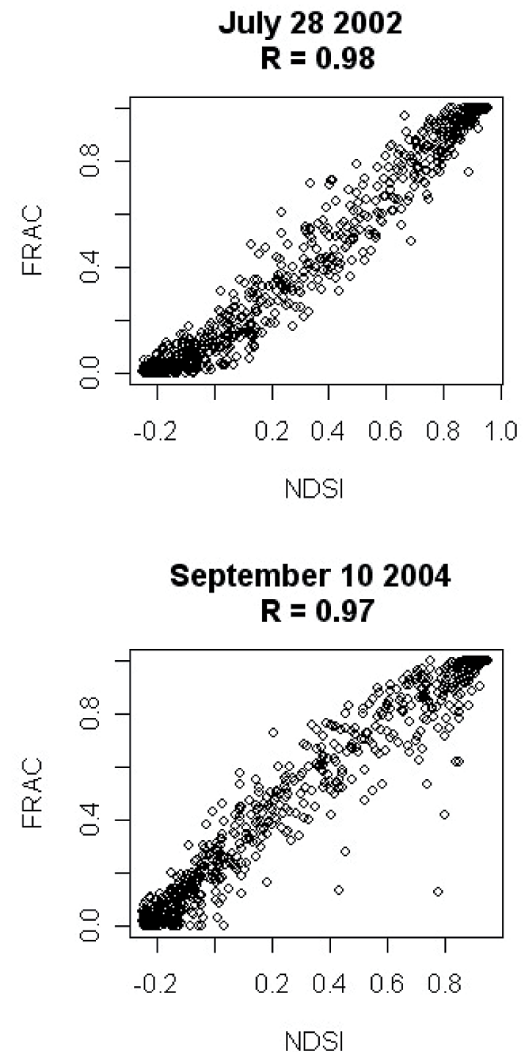

gewonnen wurde. Für jeden dieser Schnitte wird in der Folge der Korrelationskoeffizient zwischen dem Anteil der schneebedeckten Teilfläche im MODIS-Pixel und dem entsprechenden Wert des NDSI im MODIS-Pixel ermittelt. Der Anteil der schneebedeckten Teilfläche wird durch Aggregation der binären Schneekartierung (Schnitt im 3D-Modell) über das Sichtfeld (GIFOV) des jeweiligen MODIS-Pixels mit Gewichtung gemäß der MODIS-PSF (siehe Abschnitte 6.1 bis 6.3) berechnet. Danach wird derjenige Schnitt im 3D-Modell ausgewählt, welcher die höchste Korrelation zwischen MODIS NDSI und den aggregierten (Teil)Schneeflächen aufweist. Die Grenzlinien der Schneebedeckung in diesem Schnitt mit der höchsten Korrelation entsprechen dem (synthetischen) hochauflösenden Ausaperungsmuster für das jeweilige Datum der MODIS-
Aufnahme.

Die Einbeziehung zusätzlicher Information wie beispielsweise Geländehöhe und Exposition, Wind- oder Einstrahlungsverhältnisse könnte die Qualität der Interpolation bzw. des Schneehöhenmodells verbessern.

Zur Demonstration der Genauigkeitssteigerung durch Ermittlung der präzisen Sichtfelder der einzelnen Pixel und Berücksichtigung der Point Spread Function wurde eine Reihe hochauflösender Schneekartierungen, abgeleitet aus hochauflösenden Satellitenbilddaten (Landsat, Aster), verwendet. Die Ergebnisse der linearen Korrelationsanalyse für das Einzugsgebiet Mittersill sind in Abbildung 6 dargestellt. Die Graphen zeigen eine sehr hohe Korrelation zwischen NDSI aus MODIS mit exakter Geolocation (unter Berücksichtigung der PSF) und Anteil der schneebedeckten Fläche (FRAC). Allerdings variieren die Parameter der Regressionsgeraden zwischen den Aufnahmezeitpunkten. Die Gründe dafür sind möglicherweise MODIS-Sichtwinkel-Effekte und unterschiedliches Reflexionsverhalten von trockenem und nassem bzw. frischem Schnee und Firn. Im Unterschied dazu zeigt Abb. 7 die Korrelation zwischen NDSI aus dem Standard MODIS Product und dem Anteil der schneebedeckten Fläche (FRAC). Ein Vergleich der Abbildungen 6 und 7 zeigt, dass die Korrelation zwischen NDSI und anteilsmäßiger Schneefläche unter Anwendung der präzisen Verortung und der Point Spread Funktion wesentlich höher ist.

Die Abbildungen 8 und 9 illustrieren das Ergebnis der Kartierung von Schneebedeckungsmustern aus MODIS mit Subpixelauflösung. Abb. 9 zeigt auch die Grenzen dieses Verfahrens: Es können sich (nach bisherigen Erfahrungen in seltenen Fäl- 


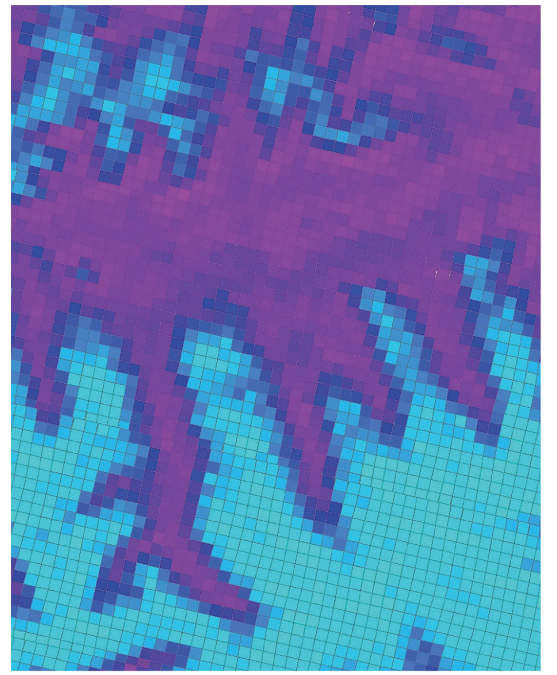

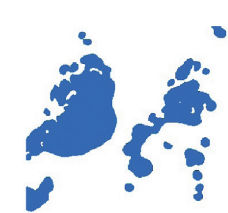

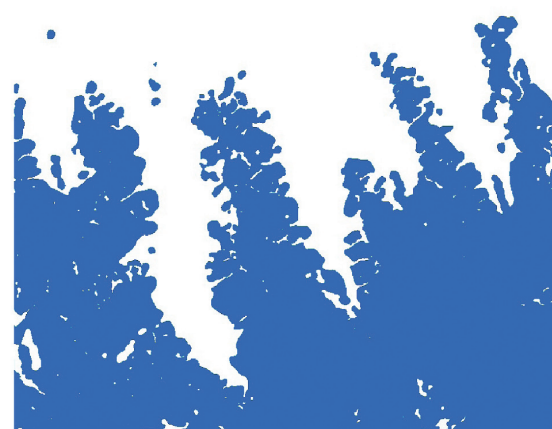

Abb. 8: Testgebiet Mittersill: MODIS Schneebedeckungskarte vom 8.6. 2004 mit exakter Geolocation (links) und daraus abgeleitete hochauflösende Schneebedeckungskarte. Der Schneeanteil pro Pixel in der MODIS Schneebedeckungskarte ist mit einer Farbskala hellviolett (kein Schnee) dunkelviolett - dunkelblau - hellblau (volle Schneebedeckung) dargestellt.
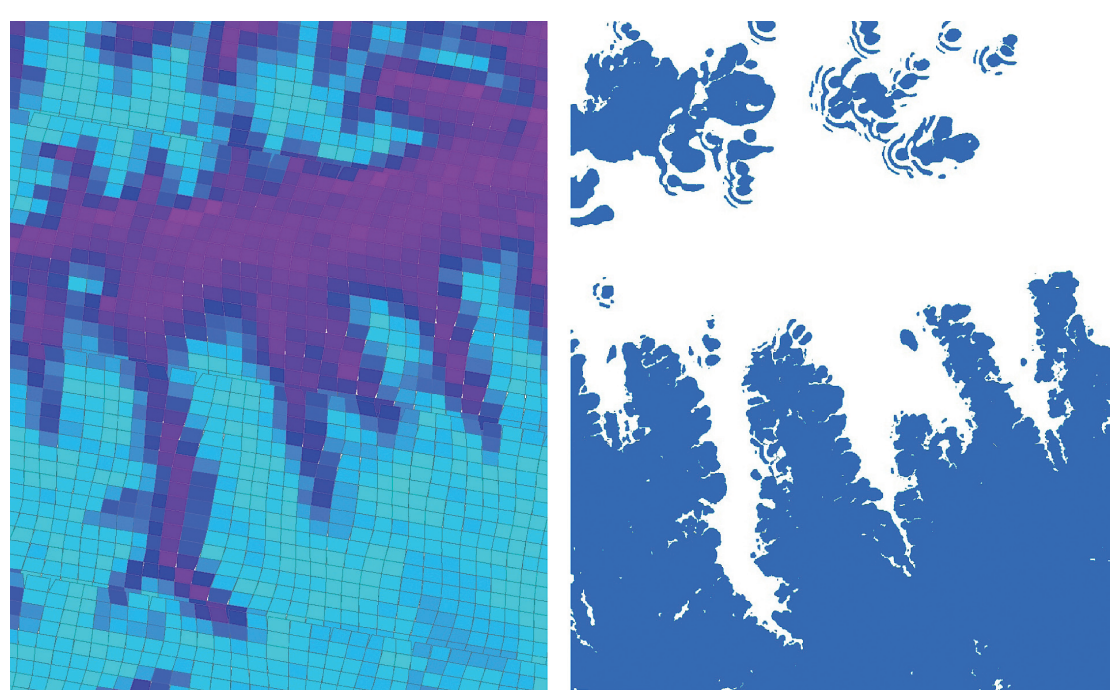

Abb. 9: Testgebiet Mittersill: MODIS Schneebedeckungskarte vom 18.5.2004 mit exakter Geolocation (links) und daraus abgeleitete hochauflösende Schneebedeckungskarte. Insbesondere in der oberen Bildhälfte sind in der hochauflösenden Karte Artefakte zu erkennen, die durch Inkonsistenzen des Schneebedeckungsmodells in diesem Bereich zu erklären sind (einander schneidende Umrisslinien schneebedeckter Flächen von unterschiedlichen Aufnahmezeiten der verwendeten hochauflösenden Satellitenbilder). Der Schneeanteil pro Pixel in der MODIS Schneebedeckungskarte ist mit einer Farbskala hellviolett (kein Schnee) - dunkelviolett - dunkelblau hellblau (volle Schneebedeckung) dargestellt. len) Artefakte in den generierten hochauflösenden Bildern zeigen, wenn die vorausgesetzte zeitliche Konstanz der Ausaperungsmuster in einem Gebiet nicht gegeben ist. Dieses Phänomen bedarf noch einer genaueren Untersuchung. Es sollte möglich sein, mit einem optimierten Interpolationsverfahren bei der Generierung des Schneebedeckungsmodells auch für diesen Fall brauchbare, wenn auch weniger genaue Schneebedeckungsmuster zu erhalten.

\section{Zusammenfassung}

Es konnte gezeigt werden, dass MODIS-Satellitenbilddaten mit ihrer hohen zeitlichen Auflösung für die Kartierung der Schneebedeckung auch in kleinen Einzugsgebieten verwendet werden können, wenn sie mit Hilfe der Geolocation-Information exakt georeferenziert werden. Unter Verwendung eines aus hochauflösenden Satellitenbildern hergestellten Schneebedeckungsmodells können auch räumlich hochauflösende Schneekarten aus MODIS-Daten hergestellt werden.

1 Institut für Vermessung, Fernerkundung und Landinformation, Universität für Bodenkultur 2 riocom - Technisches Büro für Kulturtechnik und

\section{Korrespondenz:}

Georg Kaiser

Institut für Vermessung, Fernerkundung und

Landinformation, Universität für Bodenkultur Wien E-Mail: georg.kaiser@boku.ac.at

\section{LITERATUR}

GSFC: http://modis.gsfc.nasa.gov/data/dataprod/index.php (17.9.08)

Hall DK, Martinec J (1985) Remote Sensing of Ice and Snow. Chapman and Hall, $189 \mathrm{p}$

Hall K, Riggs GA, Salomonson, VV (2001) Algorithm Theoretical Basis Document (ATBD) for the MODIS Snow //modis-snow-ice. and Sea lce-Mapping Algorithms, http://modis

Kaiser G, Schneider W (2008) Estimation of sensor Kaiser G, Schneider W (2008) Estimation of sensor tional Journal of Remote Sensing $29(7)$ p $2137-2155$
Klein AG, Hall DK, Riggs GA (1998) Improving snow cover mapping in forests through the use of a canopy reflectance model. Hydrological Processes 12(10-11) $1723-1744$

NSIDC: http://nsidc.org/data/modis/data.html (Zugriff 17.9.08)

Riggs GA, Hall DK, Salomonson VV (2006) MODIS Snow Products User Guide to Collection 5 , http://no Snow Products User Guide to Collection 5, http://mo (Zugriff 14.9.2008) Salom

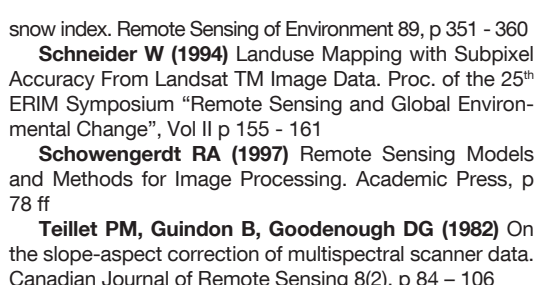

ho 